\title{
An explicit-implicit finite element model for the numerical solution of incompressible Navier-Stokes equations on moving grids
}

\author{
J. Marti ${ }^{\mathrm{a}, \mathrm{b}}$, P.B. Ryzhakov ${ }^{\mathrm{a}}$ \\ ${ }^{a}$ Centre Internacional de Mètodes Numèrics en Enginyeria (CIMNE) \\ Gran Capitán s/n, 08034 Barcelona, Spain \\ ${ }^{b}$ Department of Civil and Environmental Engineering, Universitat Politècnica de \\ Catalunya (UPC), 08034 Barcelona, Spain
}

\begin{abstract}
In this paper an efficient mesh-moving Finite Element model for the simulation of the incompressible flow problems is proposed. The model is based on a combination of the explicit multi-step scheme (Runge-Kutta) with an implicit treatment of the pressure. The pressure is decoupled from the velocity and is solved for only once per time step minimizing the computational cost of the implicit step. Novel solution algorithm alleviating time step restrictions faced by the majority of the former Lagrangian approaches is presented. The method is examined with respect to its space and time accuracy as well as the computational cost. Two numerical examples are solved: one involving a problem on a domain with fixed boundaries and the other one dealing with a free surface flow. It is shown that the method can be easily parallelized.
\end{abstract}

Keywords: incompressible Navier-Stokes, accuracy, Particle Finite Element Method, Lagrangian, OpenMP, benchmark

\section{INTRODUCTION}

Lagrangian models for the simulation of flow problems have been developed by several groups worldwide and successfully applied to a variety of engineering problems over past three decades. They were found advantageous for problems involving moving domain boundaries $[1,2,3,4,5]$

Email address: pryzhakov@cimne.upc.edu (P.B. Ryzhakov)

Preprint submitted to Computer Methods in Applied Mechanics and EngineeringMarch 8, 2019 
and interfaces, particularly, free-surface flows interacting with solid bodies $[6,7,8,9,10,11]$. They have been also applied to the simulation of different material forming processes $[4,12,13,14,15]$. Lagrangian methods are advantageous for such problems due to the intrinsic capability of tracking the boundary evolution. Unlike their fixed-grid counterparts, they do not suffer numerical diffusion and preserve interfaces sharp. Nevertheless, Lagrangian flow models are generally characterized by higher computational costs due to time step restrictions and necessity of mesh re-generation [16].

The main distinguishing feature of these approaches originates from the fact that when written in the Lagrangian framework, the governing equations of the continuum (incompressible Navier-Stokes equations in our case) become "split" into a geometrical part (tracking the motion of the particles) $d \mathrm{x} / d t=\mathrm{u}(\mathrm{x}, t)$ and a physical part (calculating how the flow variables change in time at each particle). Consequently, convective term disappears from the momentum equation of the flow, leading to several benefits: no necessity of stabilization for convection-dominated flows and symmetry of the governing system. However, the difficulty arises due to the necessity of finding an optimal way of moving the particles and coupling the particle movement to the solution of the momentum-continuity equations system. Thus, despite of the fact that the convective term vanishes (source of non-linearity in classical fixed-grid approaches), the problem remains non-linear as the fluid stresses depend on both the velocity and the position of the particles, and this latter one, in turn, depends on the velocity. This means that in order to solve the problem numerically, an iterative method is needed. All iterative methods are based on a linearization of the equations and also require an initial "guess" for starting the iterations. Depending on the way the particle movement is modeled and coupled to the solution of Navier-Stokes equations, different versions of the Lagrangian models can be distinguished.

In this work we shall concentrate on the class of Lagrangian methods known as Particle Finite Element Methods (PFEM), which are very similar to the classical Lagrangian Finite Element method used in solids. It treats the Lagrangian mesh nodes as immaterial particles, i.e. they follow the convective velocity but do not have any mass associated to them. Other popular Lagrangian methods such as Smooth Particle Hydrodynamics [17, 9,6] and Material Points Methods (MPM) [18] (where particles are treated as true material points with associated mass) lie outside the scope of the present work.

Majority of the existing Lagrangian finite element-based fluid models are 
relying on fully implicit time integration schemes [19, 16, 8, 20, 11, 21, 22, 23]. According to these schemes, the domain where equations need to be solved corresponds to the mesh defined by the nodal (particle) positions which are unknown. Typically, the particle positions are predicted using the last known value of the velocity. The overall solution procedure consists in a non-linear loop, where at each step the Navier-Stokes equations are solved and then the nodal positions are updated according to newly obtained velocity value.

Unfortunately, in all the above-mentioned mentioned approaches, the possibility of element inversion at any non-linear iteration step introduces an important limitation. Element inversion (negative Jacobians) leads to an immediate failure of the implicit solver [24]. Thus, all the mentioned approaches require estimating a critical time step in order to ensure that no element gets inverted. In practice, this may introduce a prohibitively strict time step restrictions.

In order to alleviate the above-mentioned drawbacks of the iterative procedure involved in an implicit solution Idelsohn et al [25] proposed a scheme, where the particles (nodes) were moved only once, prior to solving the NavierStokes equations. Such mesh movement step was fully explicit. The obtained configuration was considered to be the end-of-step configuration and was not further updated. In order to ensure improved accuracy of this prediction, the particles positions were obtained following the streamline corresponding to velocity at the known time step. The advantage of this method was that it allowed using large time steps without the danger of the element inversion as the mesh was considered fixed within an implicit step. Unfortunately, the accuracy of the proposed scheme resulted to be of the first order in time.

In the present work we strive to develop an alternative explicit-implicit (semi-explicit) scheme, where domain configuration is predicted in a more accurate way, while the computational cost of the implicit step is further reduced. This is achieved by using the fourth order Runge-Kutta scheme for integrating both the velocity and the particle positions. Due to the implicit nature of the pressure in incompressible flows, pressure is integrated implicitly. However, in order to reduce the computational cost of the overall method, we propose a methodology that requires solving the implicit pressure equation only once per time step and not at each sub-step of the Runge-Kutta scheme. Particular attention is given to the mesh update strategy. A technique for ensuring that element inversion does not lead to time steps limitation is proposed.

The paper is structured as follows: first, the system of governing equa- 
tions is presented. Then these are discretized in time and space. A combined explicit-implicit time integration scheme is derived. Overall solution algorithm is outlined, emphasizing the steps essential for minimizing the overall computational cost. The paper concludes with two numerical examples: one involving a problem on a domain with fixed boundaries and the other one involving a free surface flow problem. The method is examined with respect to space and time accuracy and computational cost. We note that there exist nearly no study where temporal and spatial accuracy of a Lagrangian finite element method for the fluid problems have been analyzed.

\section{EQUATIONS OF MOTION}

\subsection{Governing equations at continuum level}

Let $\Omega^{t}$ denote a domain containing a viscous incompressible fluid defined by the particles position $\mathbf{x}_{i}=\left.\chi\left(\mathbf{X}_{i}, t\right)\right|_{i=1} ^{d}$ in the Cartesian spatial coordinates

at time $t$ ( $d$ is the spatial dimension), where $\mathbf{X}$ is the position vector in the reference configuration. The knowledge of the deformation map $\chi$ for all the particles completely defines the motion and deformation of a continuum body. The evolution of the velocity $\mathbf{v}=\mathbf{v}(\mathbf{x}, t)$, the pressure $p=p(\mathbf{x}, t)$ and the position of a given material particle is governed by the following equations:

$$
\begin{gathered}
\frac{D \mathbf{x}}{D t}=\mathbf{v} \\
\frac{D \mathbf{v}}{D t}=-\frac{1}{\rho} \nabla p+\frac{1}{\rho} \nabla \cdot\left(2 \mu \frac{\nabla \mathbf{v}+\nabla^{T} \mathbf{v}}{2}\right)+\mathbf{b} \\
\nabla \cdot \mathbf{v}=0
\end{gathered}
$$

where $D \phi / D t$ represents the total or material time derivative of a function $\phi, \mu$ is the fluid viscosity, $\rho$ is the density, and $\mathbf{b}$ is the body force.

At any time $t$ Eqs. (2) and (3) can be solved on the whole volume $\Omega^{t}$ occupied by the material body, specifying the surface forces $\overline{\mathbf{t}}$ at certain surfaces $\Gamma_{\sigma}$ of the boundary $\Gamma$ (Neumann boundary condition) of the body and prescribing velocity $\overline{\mathbf{v}}$ in the remaining parts $\Gamma_{\mathbf{v}}$ of the material's boundary (Dirichlet boundary condition):

$$
-\nabla p \cdot \mathbf{n}+\nabla \cdot\left(2 \mu \frac{\nabla \mathbf{v}+\nabla^{T} \mathbf{v}}{2}\right) \cdot \mathbf{n}=\overline{\mathbf{t}} \quad \text { on } \quad \Gamma_{\sigma}
$$




$$
\mathbf{v}=\overline{\mathbf{v}} \text { on } \Gamma_{\mathbf{v}}
$$

In the following section, the temporal and spatial discretization will be presented.

\subsection{Temporal integration}

In order to integrate the governing equations of the incompressible flow in time, fully implicit schemes are the most commonly used. Thus, a non-linear system given by the momentum and continuity equations is solved iteratively at every time step providing the increments of the velocity and the pressure. While some authors apply coupled velocity-pressure (monolithic solvers) [11], most often, algorithms like fractional-step (pressure segregation) [26, 27] are used to decouple the pressure from the velocity making the solution procedure less computationally expensive. This method is used in several Lagrangian fluid models, such as e.g. [28, 19, 29, 30]. Alternatively, quasi-incompressible approaches that introduce artificial compressibility into the fluid are also employed [21, 20, 31]. These approaches also allow decoupling the velocity and the pressure solution steps.

Fully explicit methods for incompressible flows cannot be efficiently used due to the implicit nature of the pressure (a fully explicit method would be governed by the acoustic pressure scale introducing prohibitively small time step restrictions). However, recently explicit approaches with artificial compressibility have been proposed leading to somewhat improved time step estimates [32]. However, the stable time steps are directly dependent on the artificial compressibility constant and thus for obtaining reasonably large time step estimates one would need to strongly violate incompressibility constraint.

Combined explicit-implicit (semi-explicit) methods that rely on integrating velocity explicitly while treating the pressure implicitly have been proposed by several authors in the framework of fixed grid (Eulerian) approaches. These methods usually use multi-step Runge-Kutta schemes for the velocity integration. In finite volumes context this was followed e.g. in [33]. In finite difference framework this was done e.g. in [34] where a combination of 3rdorder Runge-Kutta scheme for the convective term with a Crank-Nicholson integration for the viscous term was proposed. In [35] a 4th-order RungeKutta scheme equipped with solution of pressure Poisson's equation at each sub-step was developed. The above-mentioned semi-explicit finite volume 
and finite differences approaches rely on computing the pressure at every sub-step of the multi-step scheme, which is a computationally intensive option. An idea of reducing the number of implicit steps of an explicit-implicit scheme has been proposed in [36] for a finite difference model. Similar idea was followed in the for the fixed-grid finite element models in [37] and [38].

In the present work, an explicit-implicit (semi-explicit) model based on the combination of a 4th-order Runge-Kutta with an implicit pressure integration is derived in a Lagrangian framework for the first time. In comparison with similar Eulerian models the additional challenges arise due to the fact that in the Lagrangian framework the nodes move. An additional differential equation describing the movement of the nodes must be added. In the present approach this equation is integrated numerically using the same scheme as the one used for the velocity, namely the Runge-Kutta. Nodal positions are updated at every intermediate step of the Runge-Kutta scheme. As a consequence of the nodal movement, the elemental domains (and corresponding discrete operators) are updated at each intermediate step of the Runge-Kutta scheme. Minimization of the computational cost of the method is achieved by deriving a strategy that requires solving the implicit problem (pressure) only once per time step. This is done via the application of the fractional step method. The formulation details are presented below.

For the momentum Eq.(2), the Runge-Kutta scheme yields the following time-discrete momentum equation residual [39]:

$$
\frac{\mathbf{v}^{n+1}-\mathbf{v}^{n}}{\Delta t}=\frac{1}{6}\left(\mathbf{r}_{1}+2 \mathbf{r}_{2}+2 \mathbf{r}_{3}+\mathbf{r}_{4}\right)
$$

where $\mathbf{r}_{1}, \mathbf{r}_{2}, \mathbf{r}_{3}$ and $\mathbf{r}_{4}$ are the intermediate residuals that are computed according to the formulae (note that the superindex $\beta_{i}$ distinguishes the value at the corresponding sub-step $i$ ):

FIRST RESIDUAL:

$$
\begin{aligned}
\mathbf{r}_{1}=\frac{1}{\rho}\left[-\nabla p^{n}+\nabla\right. & \left.\cdot\left(2 \mu \frac{\nabla \mathbf{v}^{n}+\nabla^{T} \mathbf{v}^{n}}{2}\right)+\rho \mathbf{b}\right] \\
\mathbf{v}^{\beta_{1}} & =\mathbf{v}^{n}+\frac{\Delta t}{2} \mathbf{r}_{1} \\
\mathbf{x}^{\beta_{1}} & =\mathbf{X}^{n}+\frac{\Delta t}{2} \mathbf{v}^{n}
\end{aligned}
$$


SeCOND Residual:

$$
\begin{gathered}
\mathbf{r}_{2}=\frac{1}{\rho}\left[-\nabla p^{\beta_{1}}+\nabla \cdot\left(2 \mu \frac{\nabla \mathbf{v}^{\beta_{1}}+\nabla^{T} \mathbf{v}^{\beta_{1}}}{2}\right)+\rho \mathbf{b}\right] \\
\mathbf{v}^{\beta_{2}}=\mathbf{v}^{n}+\frac{\Delta t}{2} \mathbf{r}_{2} \\
\mathbf{x}^{\beta_{2}}=\mathbf{X}^{n}+\frac{\Delta t}{2} \mathbf{v}^{\beta_{1}}
\end{gathered}
$$

THIRD RESIDUAL:

$$
\begin{gathered}
\mathbf{r}_{3}=\frac{1}{\rho}\left[-\nabla p^{\beta_{2}}+\nabla \cdot\left(2 \mu \frac{\nabla \mathbf{v}^{\beta_{2}}+\nabla^{T} \mathbf{v}^{\beta_{2}}}{2}\right)+\rho \mathbf{b}\right] \\
\mathbf{v}^{\beta_{3}}=\mathbf{v}^{n}+\Delta t \mathbf{r}_{3} \\
\mathbf{x}^{\beta_{3}}=\mathbf{X}^{n}+\Delta t \mathbf{v}^{\beta_{2}}
\end{gathered}
$$

FOURTH RESIDUAL:

$$
\mathbf{r}_{4}=\frac{1}{\rho}\left[-\nabla p^{\beta_{3}}+\nabla \cdot\left(2 \mu \frac{\nabla \mathbf{v}^{\beta_{3}}+\nabla^{T} \mathbf{v}^{\beta_{3}}}{2}\right)+\rho \mathbf{b}\right]
$$

Note that it is assumed that the body force $\mathbf{b}$ does not vary in time. In case of a variable body force one must update it at every intermediate step. However, this does not pose any additional difficulty as the body force is an input data.

After evaluating each residual one has to update the velocity and the particle positions as well as the fluid pressure. Eqs.(10,13,16) contain unknown pressures $p^{\beta_{1}}, p^{\beta_{2}}$ and $p^{\beta_{3}}$. Computing these pressures would require solving the Poisson's equation at every intermediate step, which is computationally expensive. Thus, we propose to evaluate the intermediate residuals $\mathbf{r}_{2}$ and $\mathbf{r}_{3}$ using the historical pressure value $\left(p^{\beta_{1}}=p^{\beta_{2}}=p^{n}\right)$, while using $p^{\beta_{3}}=p^{n+1}$ in the last one (Eq.(16)). Consequently, the equation for the velocity at time $\mathbf{v}^{n+1}$ can be written as

$$
\mathbf{v}^{n+1}=\mathbf{v}^{n}+\frac{\Delta t}{6}\left(\mathbf{r}_{1}+2 \mathbf{r}_{2}+2 \mathbf{r}_{3}+\hat{\mathbf{r}}_{4}\right)-\frac{\Delta t}{6 \rho} \nabla p^{n+1}
$$


where $\hat{\mathbf{r}}_{4}=\frac{1}{\rho}\left[\nabla \cdot\left(2 \mu \frac{\nabla \mathbf{v}^{\beta_{3}}+\nabla^{T} \mathbf{v}^{\beta_{3}}}{2}\right)+\rho \mathbf{b}\right]$ (note that $\hat{\mathbf{r}}_{4}$ contains only the effect of viscous and body forces).

Following the idea of the fractional step approach [26], the momentum equation is split into two parts by introducing the intermediate velocity $\tilde{\mathbf{v}}$.

Adding and subtracting $\tilde{\mathbf{v}}$ to the above equation and performing a second order split[40], Eq.(17) can be written as

$$
\begin{gathered}
\tilde{\mathbf{v}}=\mathbf{v}^{n}+\frac{\Delta t}{6}\left(\mathbf{r}_{1}+2 \mathbf{r}_{2}+2 \mathbf{r}_{3}+\hat{\mathbf{r}}_{4}\right)-\frac{\Delta t}{6 \rho} \nabla p^{n} \\
\mathbf{v}^{n+1}=\tilde{\mathbf{v}}+\frac{\Delta t}{6 \rho}\left(-\nabla p^{n+1}+\nabla p^{n}\right)
\end{gathered}
$$

Eq.(18) will be referred to as the "fractional momentum" and Eq.(19) will be called the "end-of-step momentum" (or "projection") equation.

The Poisson's equation for the pressure is obtained by applying the incompressibility condition(Eq.(3)) to Eq.(19), leading to

$$
\nabla \cdot \mathbf{v}^{n+1}=\nabla \cdot \tilde{\mathbf{v}}+\nabla \cdot\left[\frac{\Delta t}{6 \rho}\left(-\nabla p^{n+1}+\nabla p^{n}\right)\right]
$$

\subsection{Spatial discretization.}

For each mesh configuration (that changes not only at every time step, but also at every sub-step) we define the approximation for the components of the velocity field and the pressure using the FEM shape functions as

$$
\begin{gathered}
p(\mathbf{x})=\mathbf{N}^{T}(\mathbf{x}) p \\
\mathrm{v}_{i}(\mathbf{x})=\mathbf{N}^{T}(\mathbf{x}) \mathrm{v}_{i}
\end{gathered}
$$

where $\mathrm{v}_{i}$ represent the $\mathrm{i}$ component of the velocity field at time $\mathrm{t}$ and $\mathbf{N}^{\mathrm{T}}$ is the transpose vector of the shape functions at the same time step. Multiplying every residual equation by a test function and integrating over the domain defined by the particles position at the $t^{n}, t^{n+1 / 2}, t^{n+1 / 2}$ and $t^{n+1}$ and integrating by parts the diffusive term, we obtain the following discrete equations:

FiRst STEP (FINDING THE VALUES OF $\mathbf{v}$ AND $\mathbf{x}$ AT $t=t_{n}+\Delta t / 2$ USING THE RESIDUAL EVALUATED AT $t=t_{n}$ ): 


$$
\begin{gathered}
\mathbf{r}_{1}=\mathbf{G}_{\left(\frac{1}{\rho}\right)} \mathbf{p}^{\mathbf{n}}-\mathbf{K}_{\left(\frac{\mu}{\rho}\right)} \mathbf{v}^{\mathbf{n}}+\mathbf{F} \\
\mathbf{v}^{\beta_{1}}=\mathbf{v}^{n}+\frac{\Delta t}{2} \mathbf{M}^{-1} \mathbf{r}_{1} \\
\mathbf{x}^{\beta_{1}}=\mathbf{X}^{n}+\frac{\Delta t}{2} \mathbf{v}^{n}
\end{gathered}
$$

Second Step (Finding the values of $\mathbf{v}$ AND $\mathbf{x}$ AT $t=t_{n}+\Delta t / 2$ USING THE RESIDUAL EVALUATED AT $\left.t=t_{n}+\Delta t / 2\right)$ :

$$
\begin{gathered}
\mathbf{r}_{2}=\mathbf{G}_{\left(\frac{1}{\rho}\right)} p^{n}-\mathbf{K}_{\left(\frac{\mu}{\rho}\right)} \mathbf{v}^{\beta_{1}}+\mathbf{F} \\
\mathbf{v}^{\beta_{2}}=\mathbf{v}^{n}+\frac{\Delta t}{2} \mathbf{M}^{-1} \mathbf{r}_{2} \\
\mathbf{x}^{\beta_{2}}=\mathbf{X}^{n}+\frac{\Delta t}{2} \mathbf{v}^{\beta_{1}}
\end{gathered}
$$

Third Step (Finding the values of $\mathbf{v}$ And $\mathbf{x}$ AT $t=t_{n+1}$ Using THE RESIDUAL EVALUATED AT $\left.t=t_{n}+\Delta t / 2\right)$ :

$$
\begin{gathered}
\mathbf{r}_{3}=\mathbf{G}_{\left(\frac{1}{\rho}\right)} p^{\beta_{2}}-\mathbf{K}_{\left(\frac{\mu}{\rho}\right)} \mathbf{v}^{\beta_{2}}+\mathbf{F} \\
\mathbf{v}^{\beta_{3}}=\mathbf{v}^{n}+\Delta t \mathbf{M}^{-1} \mathbf{r}_{3} \\
\mathbf{x}^{\beta_{3}}=\mathbf{X}^{n}+\Delta t \mathbf{v}^{\beta_{2}}
\end{gathered}
$$

Fourth Step (evaluating the Velocity at $t=t_{n+1}$ Using the RESIDUAL EVALUATED AT $\left.t=t_{n+1}\right)$ :

$$
\mathbf{r}_{4}=\mathbf{G}_{\left(\frac{1}{\rho}\right)} p^{\beta_{3}}-\mathbf{K}_{\left(\frac{\mu}{\rho}\right)} \mathbf{v}^{\beta_{3}}+\mathbf{F}
$$

Particle's position $\mathbf{x}$ at time $n+1$ can be written as

$$
\mathbf{x}^{n+1}=\mathbf{X}^{n}+\frac{\Delta t}{6}\left(\mathbf{v}^{n}+2 \mathbf{v}^{\beta 1}+2 \mathbf{v}^{\beta 2}+\mathbf{v}^{\beta 3}\right)
$$


Having discretized every residual, the discrete version of the Eqs. to solve can be written as

$$
\begin{gathered}
\tilde{\mathbf{v}}=\mathbf{v}^{n}+\frac{\Delta t}{6} \mathbf{M}^{-1}\left(\mathbf{r}_{1}+2 \mathbf{r}_{2}+2 \mathbf{r}_{3}+\hat{\mathbf{r}}_{4}\right)+\frac{\Delta t}{6} \mathbf{M}^{-1} \mathbf{G}_{\left(\frac{1}{\rho}\right)} p^{n} \\
\frac{\Delta t}{6} \mathbf{L} p^{n+1}=\frac{\Delta t}{6} \mathbf{L} p^{n}-\mathbf{D}_{\rho} \tilde{\mathbf{v}} \\
\mathbf{v}^{n+1}=\tilde{\mathbf{v}}+\frac{\Delta t}{6} \mathbf{M}^{-1} \mathbf{G}_{\left(\frac{1}{\rho}\right)}\left(p^{n+1}-p^{n}\right)
\end{gathered}
$$

with

$$
\begin{aligned}
\mathbf{K}_{\left(\frac{\mu}{\rho}\right)}=\sum_{\text {elem }} \int_{\Omega} \frac{\mu}{\rho}\left(\nabla \mathbf{N} \nabla \mathbf{N}+\nabla \mathbf{N} \nabla^{T} \mathbf{N}\right) d \Omega \\
\mathbf{M}=\sum_{\text {elem }} \int_{\Omega} \mathbf{N} \mathbf{N} d \Omega \\
\mathbf{F}=\sum_{\text {elem }} \int_{\Omega} \mathbf{N b} d \Omega \\
\mathbf{G}_{\left(\frac{1}{\rho}\right)}=\sum_{\text {elem }} \int_{\Omega} \frac{1}{\rho} \nabla \mathbf{N} \mathbf{N} d \Omega \\
\mathbf{D}_{\rho}=\sum_{\text {elem }} \int_{\Omega} \rho \mathbf{N} \nabla \mathbf{N} d \Omega \\
\mathbf{L}_{\left(\frac{1}{\rho}\right)}=\sum_{\text {elem }} \int_{\Omega} \frac{1}{\rho} \nabla \mathbf{N} \nabla \mathbf{N} d \Omega
\end{aligned}
$$

Eq.(35) must be stabilized to avoid numerical oscillation due equal order of approximation for the velocity and pressure. Many of the existing techniques can be used (e.g., Galerkin/least-squares, various variational multiscale methods(ASGS,OSS) [41, 42] or finite calculus [43]). In this present work, the ASGS method was implemented. This leads to the following stabilized form of Eq. (35):

$$
\left(\frac{\Delta t}{6} \mathbf{L}+\mathbf{L}_{\tau}\right) p^{n+1}=\frac{\Delta t}{6} \mathbf{L} p^{n}-\mathbf{D}_{\rho} \tilde{\mathbf{v}}-\mathbf{S}_{\tau} \frac{\tilde{\mathbf{v}}-\mathbf{v}^{n}}{\Delta t}+\mathbf{F}_{\tau}
$$


The operators corresponding to the stabilization terms are:

$$
\begin{aligned}
\mathbf{F}_{\tau} & =\sum_{\text {elem }} \tau \int_{\Omega} \rho \nabla \mathbf{N b} d \Omega \\
\mathbf{S}_{\tau} & =\sum_{\text {elem }} \tau \int_{\Omega} \rho \nabla \mathbf{N N} d \Omega \\
\mathbf{L}_{\tau} & =\sum_{\text {elem }} \tau \int_{\Omega} \nabla \mathbf{N} \nabla \mathbf{N} d \Omega
\end{aligned}
$$

where the stabilization coefficient $\tau$ is defined as $\tau=\left(\frac{4 \mu}{\rho h^{2}}+\frac{1}{\Delta t}\right)^{-1}$, where $h$ is the element size (see e.g. [39]).

We note that since the nodal positions (and thus elemental domains) are updated at every sub-step of the scheme, the elemental matrices and vectors defined by Eqs. (37)-(42) must be recomputed at each sub-step correspondingly.

\section{OVERALL SOLUTION STRATEGY}

Re-meshing. In Lagrangian Finite Element models the mesh follows the flow motion. When applied to fluid flow problems, mesh deformations are large and therefore re-meshing may be necessary. The main challenge consists not only in the computational cost of the re-meshing procedure, but in the fact that when the mesh distortions are large it may happen that the time step size must be reduced in the run-time in order to avoid element inversions. In practice this often leads to prohibitively small time steps. Element inversion in the context of a fully implicit method leads to an immediate failure of the solver. On the other hand, in an explicit method the error is localized. This means that even if an element is inverted, one may continue the simulation provided that an error is rectified posteriorly.

Therefore to ensure that the present explicit-implicit method does not suffer from the restrictions faced by the former fully implicit ones, we propose to perform the re-meshing not at the end of the time step, but at the end of the explicit step. This ensures that when the implicit step (solution of the Poisson's equation) is performed no inverted elements are present in the mesh. 
During the explicit step the following remedy has been implemented: in case an element was found to undergo an inversion at one of the sub-steps of the Runge-Kutta scheme, prior to re-meshing the velocity and the pressure values are recomputed at all its nodes using the values of the neighboring elements. This strategy allows working with constant time steps governed exclusively by the stability criteria (given by Courant-Friedrich-Levy (CFL) number) and not by the local mesh deformations.

The procedure implemented is explained in detail using an example shown in Fig. 1. Let us consider an element surrounded by a large red circle in Fig. 1(a). Let us consider that a large nodal velocity (designated by an error) leads it to inversion, and results in a configuration shown in Fig. 1(b). For the sake of simplicity of the explanation we shall consider that the other nodes undergo a much smaller motion.

The velocity and the pressure values at the nodes of the inverted element (see red circles in Fig. 1(c)) are erroneous. We propose to interpolate them from the corresponding patches. For instance, for the node $I$ we identify the patch (large blue circle in Fig. 1(d) indicates the connectivities) and subsequently compute the approximated value as an average of the values of the patch nodes belonging to non-inverted elements (these nodes are distinguished by green circles). For example, we compute the pressure at the node $I$ as follows: $p_{I}=\frac{1}{6} \sum_{i=1}^{6} p_{i}$ (Arabic numbers stand for the nodes of "good" elements, while Roman numbers indicate the nodes of the inverted element). The same procedure is performed for each node of the inverted element and for each primary variable (velocity and pressure).

Once the explicit step is over, the re-meshing is performed. Remeshing and boundary identification used in the present work follow standard procedure implemented in all former versions of the PFEM. Therefore, these are not discussed here and the reader is referred to e.g. $[20,19,8]$ for the corresponding details. The re-meshed configuration corresponding to the above-described case is shown in Fig. 1(e).

Solution strategy. The problem to be solved can be formulated as: "given the nodal positions, the velocity and the pressure at time step $t^{n}$, find these variables at $t^{n+1}$. The overall solution strategy according to the method proposed in the present work can be summarized as follows: 


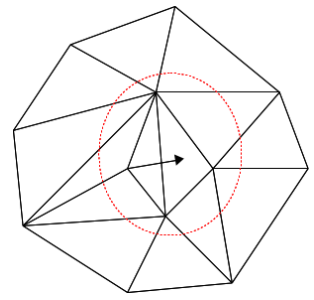

(a) Initial config.

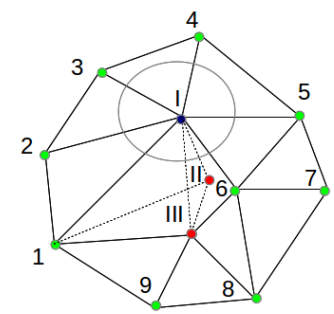

(d) Interpolation

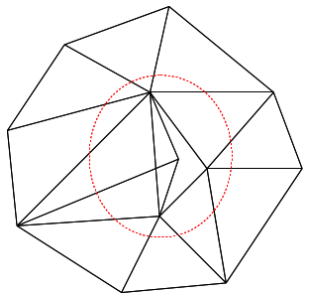

(b) Snap-through

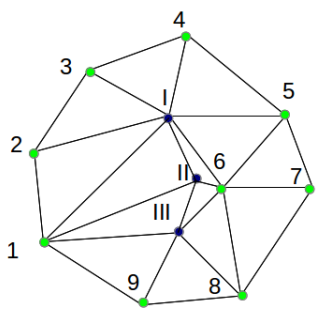

(e) After re-meshing

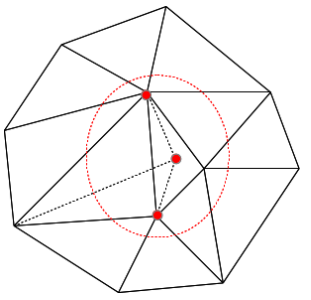

(c) Element erasing

Figure 1: Velocity fields at $\mathrm{t}=0.5 \mathrm{~s}$

\section{EXAMPLES}

The present model was implemented in Kratos Multi-Physics code, an academic Open Source software [44]. For solution of pressure Poisson's equation conjugate gradient $(\mathrm{CG})$ solver equipped with a diagonal pre-conditioner was used. The convergence tolerance was set to $10^{-6}$.

\subsection{Example with an analytic solution}

This example proposed in [40] was used in several references for assessing the accuracy and computational efficiency of incompressible flow FEM solvers. Navier-Stokes equations are solved here in a unit square domain equipped with homogeneous boundary conditions for the velocity at all the boundaries. The force vector corresponding to the following analytic solution is applied:

$$
\begin{array}{r}
\mathrm{v}_{x}(\mathrm{x}, \mathrm{y}, t)=f(\mathrm{x}) f^{\prime}(\mathrm{y}) \mathrm{g}(t) \\
\mathrm{v}_{y}(\mathrm{x}, \mathrm{y}, t)=-f^{\prime}(\mathrm{x}) f(\mathrm{y}) g(t)
\end{array}
$$

where

$$
\begin{array}{r}
f(\mathrm{x})=100 \mathrm{x}^{2}(1-\mathrm{x})^{2} \\
g(t)=\cos (4 \pi t) e^{-t}
\end{array}
$$


1. Knowing the velocity $\mathbf{v}$, pressure $p$ and nodal position $\mathbf{X}$ corresponding at time $t^{n}$ perform the explicit step:

- Evaluate $\mathbf{r}_{1}$ using Eq.(23). Find $\mathbf{v}^{\beta 1}$ and $\mathbf{x}^{\beta 1}$ (Eq.(24), Eq.(25)).

- Move particles to the new position $\mathbf{x}^{\beta 1}$.

- Update elemental matrices and vectors according to the new nodal positions (Eqs. 37-42).

- Evaluate $\mathbf{r}_{2}$ using Eq.(26). Find $\mathbf{v}^{\beta 2}$ and $\mathbf{x}^{\beta 2}$ (Eq.(27),Eq.(28)).

- Move particles to the new position $\mathbf{x}^{\beta 2}$.

- Update elemental matrices and vectors (Eqs. 37-42).

- Evaluate $\mathbf{r}_{3}$ using Eq.(29). Find $\mathbf{v}^{\beta 3}$ and $\mathbf{x}^{\beta 3}$ (Eq.(30), Eq.(31)).

- Move particles to the new position $\mathbf{x}^{\beta 3}$.

- Update elemental matrices and vectors (Eqs. 37-42).

- Find $\tilde{\mathbf{v}}$ and $\mathbf{x}^{n+1}$ solving Eq.(34) and Eq.(33), respectively.

- Move particles to the new position $\mathbf{x}^{n+1}$.

2. Check if any element was inverted at the explicit step.

- If yes: interpolate the values at the nodes of the inverted elements from the neighbors.

- If not: go to step 3.

3. Re-mesh the fluid domain (if necessary).

4. Perform the implicit step: solve the Poisson's equation for the pressure (Eq.(43)). Result: $p^{n+1}$.

5. Correct the velocity to obtain a divergence-free solution. Result: $\mathbf{v}^{n+1}$ (Eq.(36)).

6. Go to the next time step.

Table 1: Explicit-implicit solution algorithm for the incompressible flow problems simulation on a moving grid.

One of the pressure solutions satisfying the problem is

$$
p=100 \mathrm{x}^{2}
$$




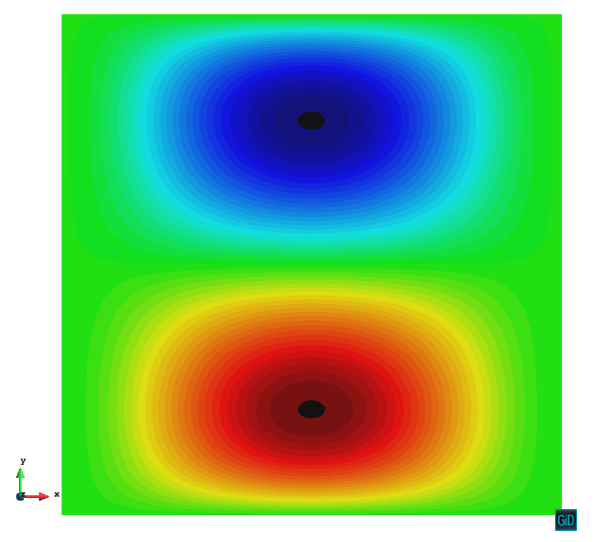

(a) $\mathrm{v}_{x}$ at t $=0.5 \mathrm{~s}$

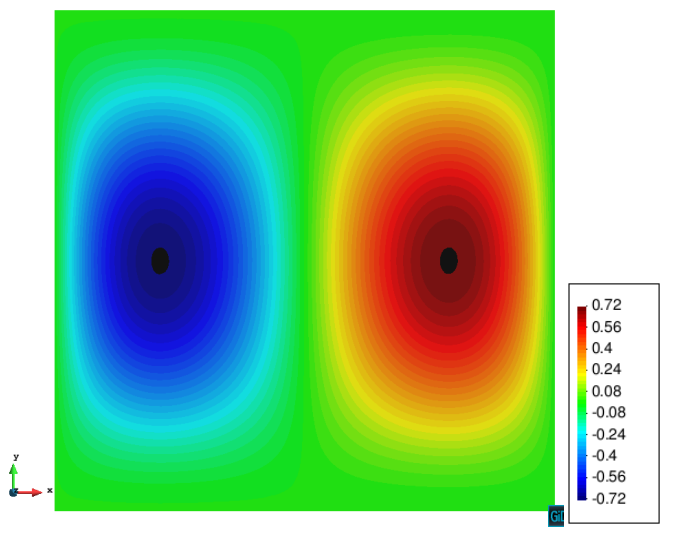

(b) $\mathrm{v}_{\mathrm{y}}$ at $\mathrm{t}=0.5 \mathrm{~s}$

Figure 2: Velocity fields at $\mathrm{t}=0.5 \mathrm{~s}$

We adopt it as an initial condition for the pressure. The force vector to be prescribed at the nodes at every step is obtained by plugging Eqs. (48) and (49) inside the momentum equation (Eq.(2)). Note that in contrast to the typical situation of force vector being the gravity (and thus constant), the body force vector in the present example depends both on time and space and therefore must be recomputed at each sub-step of the Runge-Kutta scheme.

The simulation time was set to $0.5 \mathrm{~s}$, the kinematic viscosity $\nu$ of the fluid was $0.001 \mathrm{~m}^{2} / \mathrm{s}(\nu=\mu / \rho)$ and the density $\rho=1 \mathrm{~kg} / \mathrm{m}^{3}$. The example was solved using different mesh resolutions and time step sizes in order to assess its spatial and temporal accuracy. The meshes used were structured and contained 20x20,40x40, 100x100 and 200x200 triangular elements (corresponding element sizes were $0.05,0.025,0.01$ and $0.005 \mathrm{~m}$, respectively). The time step sizes were varied from $0.0005 s$ to $0.0025 s$ using the finest mesh $(0.005 \mathrm{~m})$ in order to minimize the error in space.

Spatial accuracy. Figs. 2(a) and 2(b) display the horizontal and the vertical velocity fields at the end of the simulation $(\mathrm{t}=0.5 \mathrm{~s})$.

Fig. 3(a) shows temporal evolution of the horizontal velocity $v_{x}$ at the fixed spatial point $(\mathrm{x}, \mathrm{y})=(0.75,0.25)$. The solution corresponds to the mesh of $200 \times 200$ and time step of $0.0005 \mathrm{~s}$. Numerical and analytic solutions are superimposed and one can see that these are coincident. Fig. 3(b) provides an insight of the accuracy in terms of convection of the particles: it shows the trajectory of the particle located initially at $(0.75,0.25)$ obtained using 

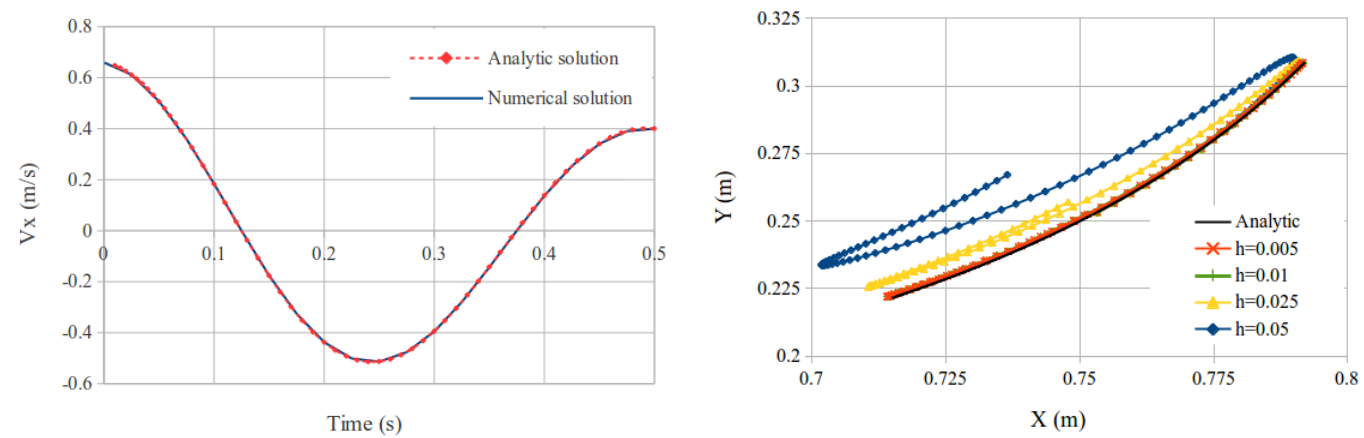

(a) Velocity evolution at fixed spatial point (b) Trajectories of the particle located at $(0.75,0.25)$

$(0.75,0.25)$ at $\mathrm{t}=0$

Figure 3: Velocity field and velocity evolution in the verification example.

different mesh sizes. One can see that for mesh size $h \leq 0.01$ the obtained trajectory is practically indistinguishable from the analytic solution.

In order to quantify the spatial accuracy of the method the final position of the particle located initially at the point $(\mathrm{x}, \mathrm{y})=(0.75,0.25)$ was recorded using different mesh resolutions (time step was set to $0.0005 s$ and the error was measured. The final location of this particle is shown in Fig. 4(a). In order to assess the effect of the re-meshing (nodal reconnection) the test was executed with and without re-meshing.

One can see that as the mesh size decreases the final location obtained using the numerical simulation is approaching the analytic result. A similar tendency (in terms of absolute value of the error) is observed with and without re-meshing. Important observation is that the re-meshing affects the result but does not affect the rate of convergence significantly and tends to the same result as the mesh size diminishes. This can be also seen in Fig. 4 (b), where the relative error versus mesh size is displayed in logarithmic scale. Error was computed as

$$
\operatorname{Err}=\frac{\sqrt{\left.\left(\mathrm{x}_{\text {num }}-\mathrm{x}_{r}\right)^{2}+\left(\mathrm{y}_{\text {num }}-\mathrm{y}_{r}\right)^{2}\right)}}{\sqrt{\mathrm{x}_{r}^{2}+\mathrm{y}_{r}^{2}}}
$$

where $\left(\mathrm{x}_{r}, \mathrm{y}_{r}\right)$ is the location of the particle according to the reference solution (analytic one, in this case) and $\left(\mathrm{x}_{\text {num }}, \mathrm{y}_{\text {num }}\right)$ are the corresponding simulation results. In both cases (with and without re-meshing) the spatial 


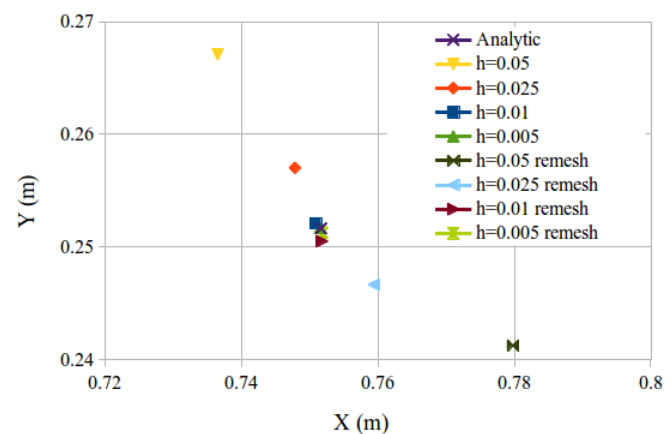

(a) Final position

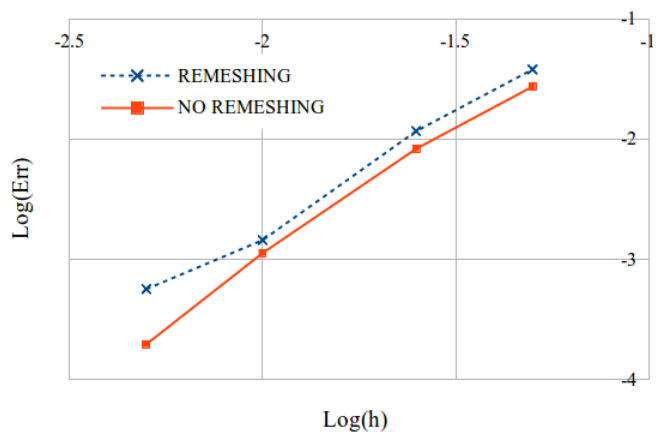

(b) Relative error

Figure 4: Final position $(\mathrm{t}=0.5 \mathrm{~s})$ of the particle initially located at $(0.25,0.75)$ obtained using different mesh resolutions.

error decreases quadratically with the mesh size $h$.

Time accuracy. Next the time accuracy is assessed. Fig. 5(a) shows the final position of the particle initially located at $(0.75,0.25)$ obtained on the mesh of $200 \times 200$ elements using 4 different time steps ranging from 0.0025 to $0.0005 \mathrm{~s}$. Since the spatial error remains constant for a given mesh and dominates for small time step sizes, in order to assess the time convergence the numerical solution obtained using an very small time step $(\mathrm{dt}=0.0001)$ was chosen as the reference solution. Thus, one avoids the dependence of the result on the spatial error. Fig. 5(b) shows the relative error in the final position versus time step size in a logarithmic scale. One can see that the convergence rate is quadratic. Fig. 6(a) shows the error in velocity computed at the considered particle. Once again, the error rate is quadratic. In the same figure we display the results obtained using a fully implicit second order backward differentiation method (BDF2) [38, 45]. One can see that the present approach shows nearly identical convergence in both the value and the slope. The error convergence rates obtained indicate that the proposed explicitimplicit scheme provides attractive convergence characteristics in spite of the fact that the pressure is solved only once per time step. Therefore, for the situations where time step estimates according to Courant-FriedrichsLevy (CFL) criterion are favorable, the proposed integration method can be very advantageous from the point of view of computational efficiency in comparison with the commonly used fully implicit schemes such as e.g. BDF2 or 


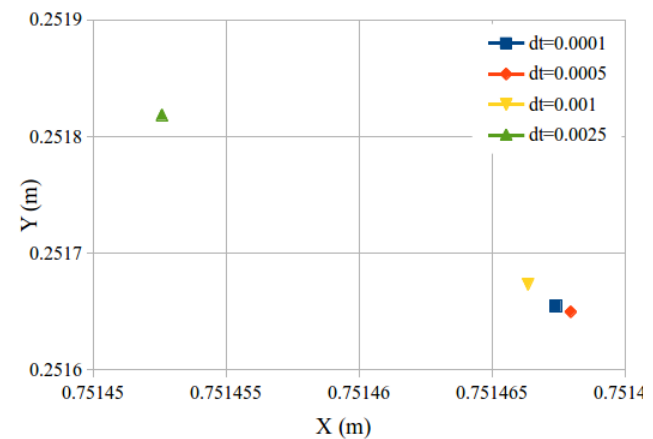

(a) Final position

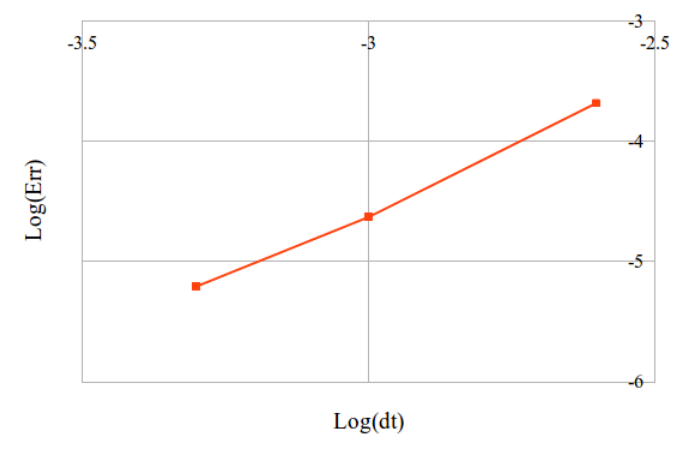

(b) Relative error

Figure 5: Final position $(\mathrm{t}=0.5 \mathrm{~s})$ of the particle initially located at $(0.25,0.75)$ obtained using different time step sizes.

Crank-Nicholson method.

Comparison of the results obtained using the present method with the ones reported in [46]. In the reference the particle positions are obtained using explicit streamline integration using the historical velocity $\mathbf{v}_{n}$ according to the method originally proposed in [47]. One can see that the rate of convergence of the present method is similar to that of the reference. However, the error itself is considerably smaller (nearly one order of magnitude) in case of using the present methodology. This suggests that, as expected, the domain configuration prediction obtained by the explicit step using Runge-Kutta scheme is more accurate than that of the streamline integration, which is done using only the historical velocity values.

Computational efficiency. Computational cost of the time integration of the present method is governed by the cost of the implicit step, which involves the solution of the Poisson's equation (if no re-meshing is performed). This is due to the fact that the explicit step is "cheaper" than the implicit step per se and, moreover, can be easily parallelized. Not pretending to present an efficiency study we provide some indications of the computational cost of the different steps of the method and the speed-up obtained when parallelizing it.

The 4 explicit steps of Runge-Kutta scheme involve assembly of global 


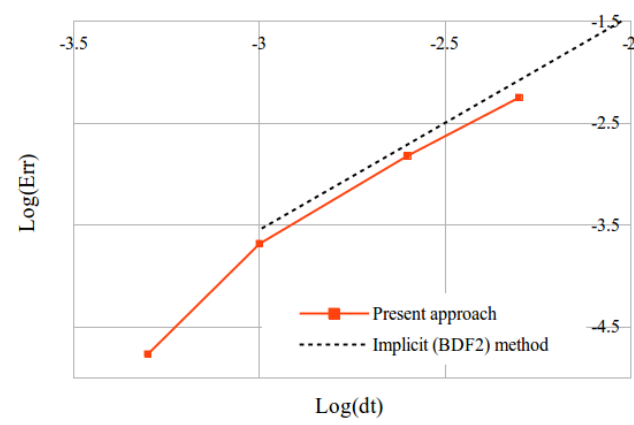

(a) Versus fixed-grid approach

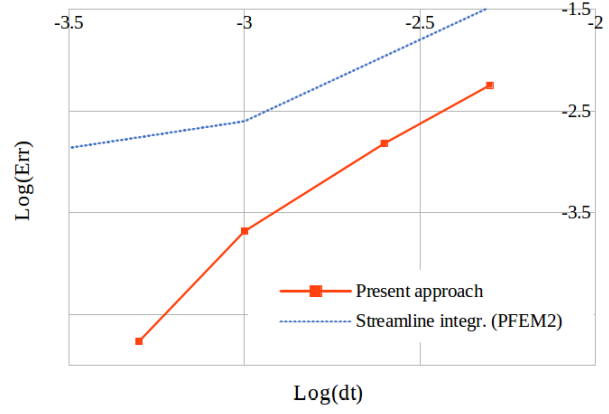

(b) Versus streamline integration

Figure 6: Relative error in the horizontal velocity at $0.5 \mathrm{~s}$. Comparison of the present approach with the former methods.

vectors (residual of the momentum equation), which involve loops over all the elements of the model. These loops were parallelized (note that the elemental contributions to the global vectors are independent among themselves) using the OpenMP library.

In order to assess the computational efficiency a cluster composed of homogeneous machines and Slurm resource scheduler were used. The used cluster node was of the class Bull bullx B510 with NUMA architecture and two processors Intel Xeon E5-2670, each one with 8 cores, 4x8 GB of RAM per CPU and four memory channels. As floating-point operations are used intensively, hyper-threading was disabled. All saving power options were also disabled. At every execution, only one processor has been used in order to avoid the use of Intels QPI, the exploitation of L3 cache and the use of the proper NUMA node memory. To do this, all executions have been launched using NUMActl to select sequentially the cores of the processor.

Table 2 shows the average computational times of the explicit and the implicit steps obtained on two meshes (one of 6000 elements and one of 300000 elements approximately). The tests were carried out using a sequential implementation and a parallel implementation. One can see that in a sequential implementation the explicit step is approximately 2.5-3 times "cheaper" than the implicit one. Parallel implementation leads to a considerable speed-up, which is, however, lower than the ideal. One can see the comparison between the ideal and the obtained speed-up of the explicit step on Fig. 7. For example, when 8 cores are used the observed speed-up is about the factor of 6 , which is satisfactory considering that the ideal speed-up would have been 


\begin{tabular}{|c||c|c|}
\hline Simulation & Expl. step & Impl. step \\
\hline Mesh1, sequential & $0.015 s$ & $0.039 s$ \\
\hline Mesh1, 8 cores & $0.0028 s$ & $0.0076 s$ \\
\hline \hline Mesh2, sequential & $0.75 s$ & $2.3 s$ \\
\hline Mesh2, 8 cores & $0.14 s$ & $0.35 s$ \\
\hline
\end{tabular}

Table 2: Computational cost of different solution steps of the proposed strategy.

around 8 .

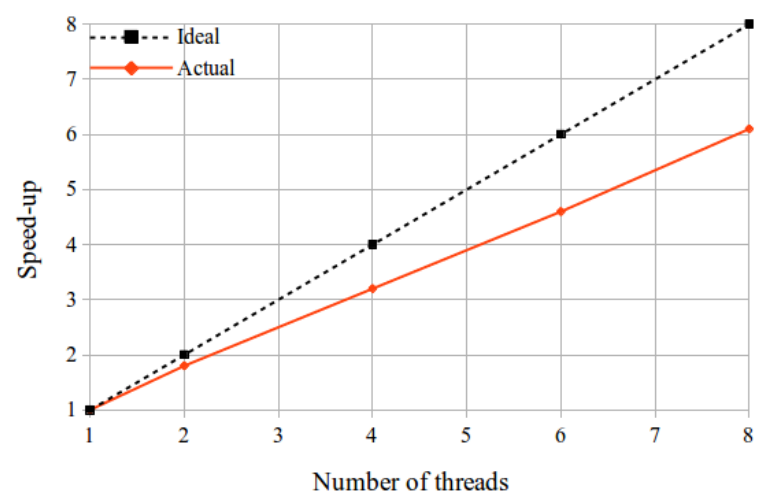

Figure 7: Speed-up obtained via OpenMP parallelization of the explicit step

\subsection{Free surface sloshing}

In this example we analyze the application of the present method to the simulation of a free-surface fluid sloshing [2]. Initial domain configuration is shown in Fig. 8(a). Homogeneous boundary condition is prescribed at all the walls of the domain. The dimensions are: $\mathrm{H}=0.1 \mathrm{~m}$ and $\mathrm{h}=0.02 \mathrm{~m}$. The free surface shape is a sinusoid. The properties are: density $\rho=1000$ $\mathrm{kg} / \mathrm{m}^{3}$, gravity $g=-9.8 \mathrm{~m} / \mathrm{s}^{2}$. The viscosity is $\nu=0.0001 \mathrm{~m}^{2} / \mathrm{s}(\nu=\mu / \rho)$ if not mentioned otherwise. A total time of $1 s$ is simulated. The domain is discretized with an unstructured triangular mesh of size $0.002 m$ (6000 elements approximately).

Fig. 8(b) shows the evolution of the free surface at the left wall of the container. The result obtained using present methodology is compared with the results obtained by using an implicit solver proposed in [48]. The implicit solver results obtained using a time step $\mathrm{dt}=0.001 s$ are taken as a reference 


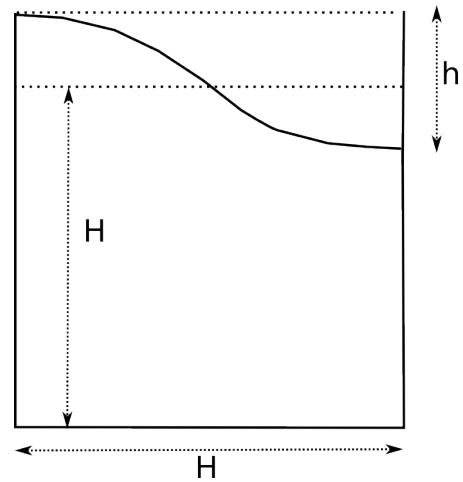

(a) Initial domain geometry

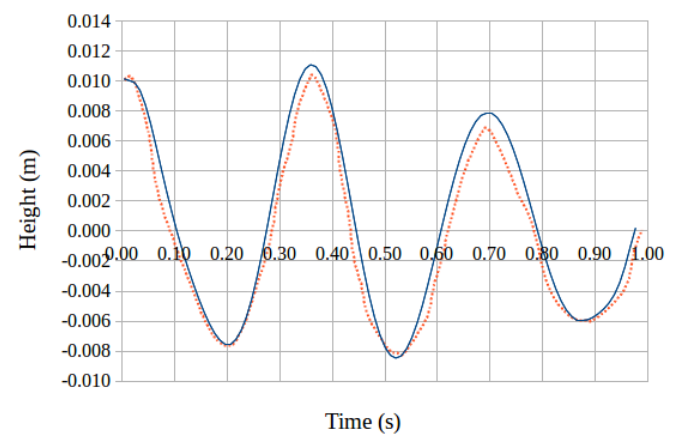

(b) Wave height at the left wall

Figure 8: Free-surface fluid sloshing example.

solution. The simulation using the present model was carried out using $\mathrm{dt}=0.005 \mathrm{~s}$. One can see a good agreement between the curves.

Fig. 9 shows the domain configuration and the corresponding velocity and pressure distribution at 3 time instances. One can see a nearly hydrostatic pressure distribution. Maximum velocity of around $0.18 \mathrm{~m} / \mathrm{s}$ is observed.

Time step size. In order to see the advantage of the strategy proposed in the present paper the example was simulated using the present approach and an implicit one [48], where mesh is moved at every non-linear iteration of the solution of the momentum equation. We note that a strategy similar to [48] is used in the majority of the Lagrangian FEM fluid models such as $[19,49,21]$. Thus, in we shall refer to this strategy as "standard".

Fig. 10(a) shows the time step sizes used throughout the simulation carried out using viscosity of $0.0001 \mathrm{~m}^{2} / \mathrm{s}$. In this case, due to relatively high viscosity, the mesh deformations are moderate. For $\mathrm{CFL}=0.5$ both the present and the standard strategies maintained the maximum time step size. For $\mathrm{CFL}=1$ the standard implicit strategy required to diminish the time step due to element deformation at several instances, while the method proposed here allowed to maintain the constant time step.

Fig. 10(b) shows the time step sizes used throughout the simulation carried out using viscosity of $0.00001 \mathrm{~m}^{2} / \mathrm{s}$. Here the mesh deformation becomes significantly larger due to lower viscosity. Thus, even at $\mathrm{CFL}=0.5$ the standard approach requires diminishing time steps on many occasions. At $\mathrm{CFL}=1$ the number of diminished time steps further increases. On the other 


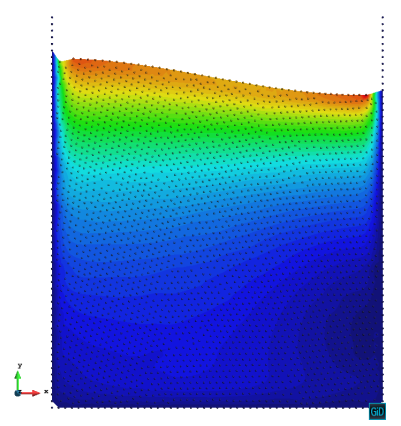

(a) $\mathbf{v}$ at $\mathrm{t}=0.055 \mathrm{~s}$

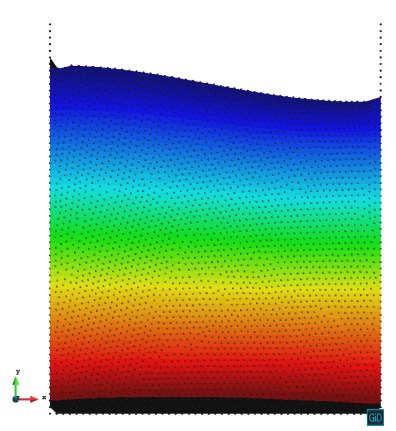

(d) $p$ at t $=0.055 \mathrm{~s}$

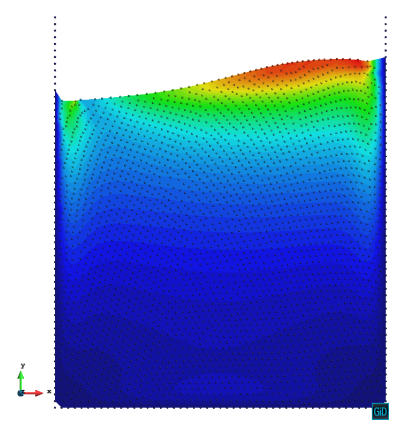

(b) $\mathbf{v}$ at t $=0.22 \mathrm{~s}$

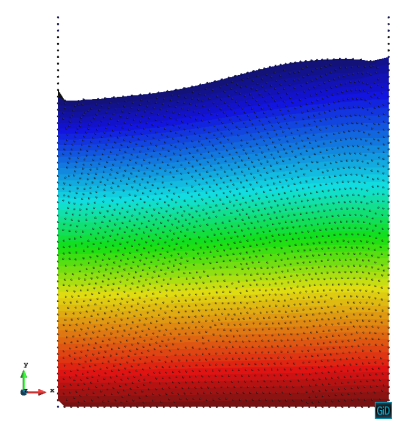

(e) $p$ at $\mathrm{t}=0.22 \mathrm{~s}$

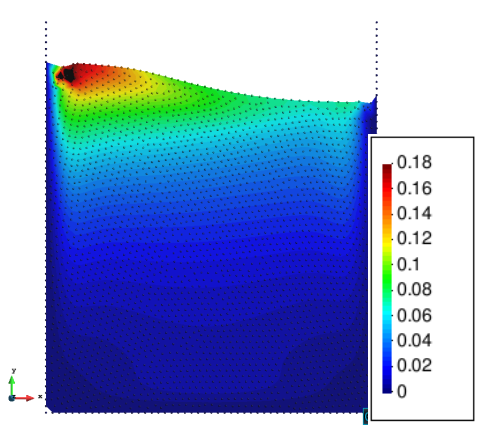

(c) $\mathbf{v}$ at $t=0.305 \mathrm{~s}$

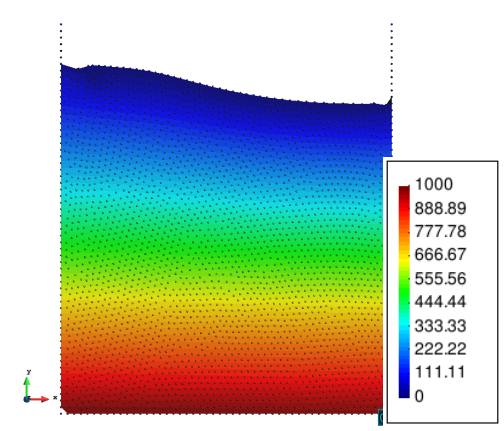

(f) $p$ at $\mathrm{t}=0.305 \mathrm{~s}$

Figure 9: Free-surface sloshing: velocity and pressure distributions at different time instances. 


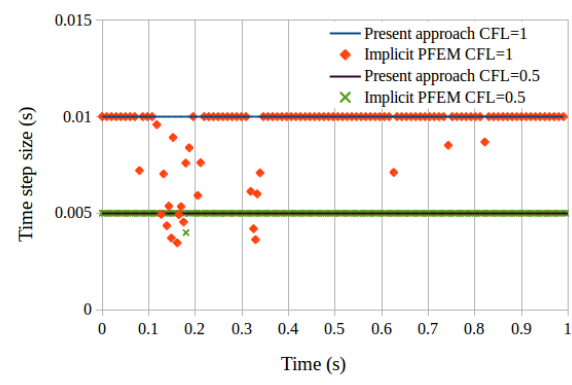

(a) Viscosity $\nu=0.0001 \mathrm{~m}^{2} / \mathrm{s}$

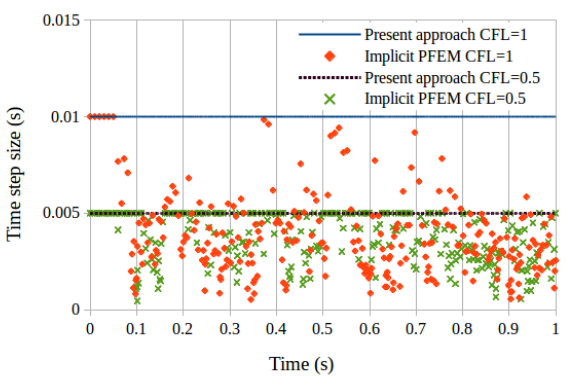

(b) Viscosity $\nu=0.00001 \mathrm{~m}^{2} / \mathrm{s}$

Figure 10: Used time step size in the simulation of a free-surface sloshing.

hand, the method proposed here maintains constant time step at both values of CFL since the implicit step is always performed on a newly generated mesh, while the mesh movement is done prior to this step explicitly.

\section{SUMMARY AND CONCLUSIONS}

In this paper we presented an explicit-implicit Lagrangian Finite Element model for the simulation of incompressible flow problems. The main idea consisted in combining an explicit multi-step scheme (Runge-Kutta) for velocity integration with an implicit integration of the pressure. We have shown that applying the fractional step approach to the governing equations one can obtain an integration scheme that requires solving the pressure Poisson's equation only once per time step. The overall solution algorithm presented here allowed working with constant time steps without the danger of inverting an element at the implicit step (which was the main problem of the majority of formerly proposed Lagrangian finite element fluid solvers).

The numerical tests carried out revealed that the proposed method is characterized by quadratic convergence in time and space. It was discovered that its time accuracy is identical with that of the commonly used implicit methods. On the other hand, the computational cost of the simulations using our model is very attractive being governed by the cost of the solution of the linear Poisson's equation for the pressure, while the formerly proposed fully implicit fractional step-based schemes additionally required costly iterative implicit solution for the velocity. It was shown that the explicit step of the method can be very easily parallelized using the OpenMP library. 
Even though the method proposed here defines a very attractive version of the PFEM for the problems where stable time step estimates are favorable, one must keep in mind that for practical purposes it still relies on reconstructing of the entire mesh. This step is generally non-parallelizable and can define a bottleneck of the model. Developing a method that optimizes the mesh re-construction (for example, by considering local re-meshing or reconnection, rather than re-constructing the entire mesh) defines the next step that must be done for establishing a new generation of efficient Lagrangian fluid solvers.

Acknowledgments. The authors also express their gratitude to Mr. A. Burgos for the help with the parallelization of the explicit solver.

[1] B. Ramaswamy and M. Kawahara. Lagrangian finite element analysis applied to viscous free surface fluid flow. International Journal for Numerical Methods in Fluids, 7(9):953-984, 1987.

[2] R. Radovitzky and M. Ortiz. Lagrangian finite element analysis of newtonian fluid flows. International Journal for Numerical Methods in Engineering, 43(4):607-619, 1998.

[3] P.B. Ryzhakov, J. Garcia, and E. Oñate. Lagrangian finite element model for the 3D simulation of glass forming processes. Computers \& Structures, 177:126-140, 2016.

[4] F. Muttin, T. Coupez, M. Bellet, and J.-L. Chenot. Lagrangian finite-element analysis of time-dependent viscous free-surface flow using an automatic remeshing technique: Application to metal casting flow. International Journal for Numerical Methods in Engineering, 36(12):2001-2015, 1993.

[5] A. Bennett. Lagrangian fluid dynamics. Cambridge University Press, 2006.

[6] C. Antoci, M. Gallati, and S. Sibilla. Numerical simulation of fluidstructure interaction by SPH. Computers \& Structures, 85(11):879-890, 2007.

[7] J. Degroote, A. Souto-Iglesias, W. Van Paepegem, S. Annerel, P. Bruggeman, and J. Vierendeels. Partitioned simulation of the interaction between an elastic structure and free surface flow. Computer 
methods in applied mechanics and engineering, 199(33):2085-2098, 2010 .

[8] E. Oñate, S.R. Idelsohn, F. Del Pin, and R. Aubry. The particle finite element method: an overview. International Journal of Computational Methods, 1:267-307, 2004.

[9] E.S. Lee, C. Moulinec, R. Xu, D. Violeau, D. Laurence, and P. Stansby. Comparisons of weakly compressible and truly incompressible algorithms for the SPH mesh free particle method. Journal of computational physics, 227(18):8417-8436, 2008.

[10] S.R. Idelsohn, E. Oñate, N. Nigro, P. Becker, and J. Gimenez. Lagrangian versus Eulerian integration errors. Computer Methods in Applied Mechanics and Engineering, 293:191-206, 2015.

[11] M. Cremonesi, A. Frangi, and U. Perego. A Lagrangian finite element approach for the analysis of fluid-structure interaction problems. International Journal for Numerical Methods in Engineering, 84(5):610630, 2010 .

[12] M. Hyre. Numerical simulation of glass forming and conditioning. Journal of the American Ceramic Society, 85(5):1047-1056, 2002.

[13] E. Oñate, J. Rojek, M. Chiumenti, S.R. Idelsohn, F. Del Pin, and R. Aubry. Advances in stabilized finite element and particle methods for bulk forming processes. Computer methods in applied mechanics and engineering, 195(48-49):6750-6777, 2006.

[14] E. Feulvarch, N. Moulin, P. Saillard, T. Lornage, and J.-M. Bergheau. $3 \mathrm{~d}$ simulation of glass forming process. Journal of materials processing technology, 164:1197-1203, 2005.

[15] P. B. Ryzhakov. An axisymmetric PFEM formulation for bottle forming simulation. Computational Particle Mechanics, 4(1):3-12, 2017.

[16] Y.T. Feng and D. Perić. A time-adaptive space-time finite element method for incompressible Lagrangian flows with free surfaces: computational issues. Computer Methods in Applied Mechanics and Engineering, 190(5-7):499-518, 2000. 
[17] J. P. Morris, P. J. Fox, and Y. Zhu. Modeling low Reynolds number incompressible flows using SPH. Journal of computational physics, 136(1):214-226, 1997.

[18] Z. Wickowski. The material point method in large strain engineering problems. Computer Methods in Applied Mechanics and Engineering, 193(39):4417 - 4438, 2004. The Arbitrary Lagrangian-Eulerian Formulation.

[19] S.R Idelsohn, E. Oñate, and F. Del Pin. The particle finite element method: a powerful tool to solve incompressible flows with free-surfaces and breaking waves. International Journal of Numerical Methods in Engineering, 61:964-989, 2004.

[20] S.R Idelsohn, J. Marti, A. Limache, and E. Oñate. Unified Lagrangian formulation for elastic solids and incompressible fluids. application to fluid-structure interaction problems via the PFEM. Computer Methods in Applied Mechanics and Engineering, 197:17621776, 2008.

[21] E. Oñate, A. Franci, and J. M. Carbonell. Lagrangian formulation for finite element analysis of quasi-incompressible fluids with reduced mass losses. International Journal for Numerical Methods in Fluids, 74(10):699-731, 2014.

[22] P.B. Ryzhakov and A. Jarauta. An embedded approach for immiscible multi-fluid problems. International Journal for Numerical Methods in Fluids, 81(6):357-376, 2016.

[23] M. Zhu and M. H. Scott. Unified fractional step method for Lagrangian analysis of quasi-incompressible fluid and nonlinear structure interaction using the pfem. International Journal for Numerical Methods in Engineering, 109(9):1219-1236, 2017.

[24] P.B. Ryzhakov, J. Marti, S.R. Idelsohn, and E. Oñate. Fast fluidstructure interaction simulations using a displacement-based finite element model equipped with an explicit streamline integration prediction. Computer Methods in Applied Mechanics and Engineering, 315:10801097, 2017.

[25] S.R. Idelsohn, J. Marti, P. Becker, and E. Oñate. Analysis of multifluid flows with large time steps using the particle finite element method. 
International Journal for Numerical Methods in Fluids, 75(9):621-644, 2014.

[26] A.J. Chorin. A numerical method for solving incompressible viscous problems. Journal of Computational Physics, 2:12-26, 1967.

[27] R. Codina. A stabilized finite element method for generalized stationary incompressible flows. Computer Methods in Applied Mechanics and Engineering, 190(20-21):2681 - 2706, 2001.

[28] M. Hayashi, K. Hatanaka, and M. Kawahara. Lagrangian finite element method for free surface Navier-Stokes flow using fractional step methods. International journal for numerical methods in fluids, 13(7):805-840, 1991.

[29] M. Zhu and M. H. Scott. Improved fractional step method for simulating fluid-structure interaction using the pfem. International Journal for Numerical Methods in Engineering, 99(12):925-944, 2014.

[30] P.B. Ryzhakov. A modified fractional step method for fluid-structure interaction problems. Revista Internacional de Métodos Numéricos para Cálculo y Diseño en Ingeniería, 33(1-2):58-64, 2017.

[31] A. Franci, E. Oñate, and J.M. Carbonell. On the effect of the bulk tangent matrix in partitioned solution schemes for nearly incompressible fluids. International Journal for Numerical Methods in Engineering, 102(3-4):257-277, 2015.

[32] M. Cremonesi, S. Meduri, U. Perego, and A. Frangi. An explicit Lagrangian finite element method for free-surface weakly compressible flows. Computational Particle Mechanics, 4(3):357-369, Jul 2017.

[33] B. Sanderse and B. Koren. Accuracy analysis of explicit Runge-Kutta methods applied to the incompressible Navier-Stokes equations. Journal of Computational Physics, 231(8):3041-3063, 2012.

[34] G.M. Fishpool and M.A. Leschziner. Stability bounds for explicit fractional-step schemes for the Navier-Stokes equations at high Reynolds number. Computers \& Fluids, 38(6):1289-1298, 2009. 
[35] N. Kampanis and J. Ekaterinaris. A staggered grid, high-order accurate method for the incompressible Navier-Stokes equations. Journal of Computational Physics, 215(2):589-613, 2006.

[36] H. Le and P. Moin. An improvement of fractional step methods for the incompressible Navier-Stokes equations. Journal of computational physics, 92(2):369-379, 1991.

[37] N. Nikitin. Third-order-accurate semi-implicit Runge-Kutta scheme for incompressible Navier-Stokes equations. International Journal for Numerical Methods in Fluids, 51(2):221-233, 2006.

[38] P.B. Ryzhakov and J. Marti. A semi-explicit multi-step method for solving incompressible Navier-Stokes equations. Applied Sciences, 8(1):119, 2018.

[39] J. Donea and A. Huerta. Finite element method for flow problems. J. Wiley edition, 2003.

[40] R. Codina. Pressure stability in fractional step finite element method for incompressible flows. Journal of Computational Physics, 170:112-140, 2001.

[41] T. J. R. Hughes. Multiscale phenomena: Green's functions, the Dirichlet-to-Neumann formulation, subgrid scale models, bubbles and the origins of stabilized methods. Computer Methods in Applied Mechanics and Engineering, 73:387-401, 1995.

[42] R. Codina. A stabilized finite element method for generalized stationary incompressible flows. Computer Methods in Applied Mechanics and Engineering, 190(20-21):2681 - 2706, 2001.

[43] E. Oñate. Possibilities of finite calculus in computational mechanics. Int. Journ. Num. Meth. Engng., 60 (1):255-281, 2004.

[44] P. Dadvand, R. Rossi, and E. Oñate. An object-oriented environment for developing finite element codes for multi-disciplinary applications. Archieves of Computational Methods in Engineering, 17/3:253-297, 2010 . 
[45] P.B. Ryzhakov, J. Cotela, R. Rossi, and E. Oñate. A two-step monolithic method for the efficient simulation of incompressible flows. International Journal for Numerical Methods in Fluids, 74(12):919-934, 2014.

[46] P. Becker. An enhanced Particle Finite Element Method with special emphasis on landslides and debris flows. PhD thesis, Universitat Politecnica de Catalunya, 2015.

[47] Idelsohn S., Nigro N., Gimenez J., Rossi R., and Marti J. A fast and accurate method to solve the incompressible Navier-Stokes equations. Engineering Computations, 30(2):197-222, 2013.

[48] P.B. Ryzhakov, E. Oñate, R. Rossi, and S.R. Idelsohn. Improving mass conservation in simulation of incompressible flows. Int. Jour. for Num.Methds. in Eng., 2012. early view, published online 29/03/2012.

[49] J. Marti, P.B. Ryzhakov, S.R Idelsohn, and E. Oñate. Combined Eulerian-PFEM approach for analysis of polymers in fire situations. Int. J. Numer. Meth. Engng., 92:782-801, 2010. 